\title{
Interrelación entre el crecimiento y la rentabilidad de la banca comercial en España*
}

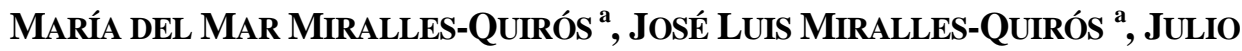 \\ DAZA-IZQUIERDO ${ }^{\text {a }}$ \\ a Universidad de Extremadura, Facultad de CC.EE. y EE., Avda. de Elvas, s/n, 06071 Badajoz, \\ España.E-mail: marmiralles@unex.es,miralles@unex.es, juliodaza@unex.es
}

\begin{abstract}
RESUMEN
En los últimos años, el sector financiero español ha sufrido importantes cambios que han afectado tanto a la estructura como a los resultados de los bancos comerciales. En este contexto, el objetivo del presente estudio consiste en analizar la interrelación entre los dos objetivos básicos de la banca comercial: el crecimiento y la rentabilidad. A diferencia de la literatura previa, en la presente investigación proponemos un análisis no lineal de la relación entre ambas magnitudes así como del efecto directo de las medidas adoptadas para el saneamiento y recapitalización de las entidades. Los principales resultados obtenidos nos indican que durante los años 2005-2014 existe una relación cuadrática entre el crecimiento y la rentabilidad de los bancos españoles y que las medidas de saneamiento y recapitalización han tenido un efecto significativo sobre el crecimiento y la rentabilidad de las entidades analizadas.
\end{abstract}

Palabras clave: Crecimiento, rentabilidad, banca comercial, datos de panel.

\section{Interrelation between Growth and Profitability in Spanish Commercial Banks}

\begin{abstract}
In recent years, the financial sector in Spain has suffered a huge change which has affected the structure and results of commercial banks. In this context, the aim of this study is to analyze the interactions between the two main objectives of commercial banks: growth and profitability. We contribute to financial literature by providing a non-linear analysis to explain the interrelationship between these two magnitudes, as well as the direct effect of the bank recapitalization and restructuring. Our overall results reveal the existence of a quadratic relationship between bank growth and profitability in Spain during the 2005-2014 period. Moreover, our findings show that the measures adopted by the Spanish authorities have taken significant effects on the analyzed magnitudes.
\end{abstract}

Keywords: Growth, Profitability, Commercial Banks, Panel Data.

Clasificación JEL: G01, G20, G21

\footnotetext{
* Este trabajo ha recibido la ayuda financiera de la Junta de Extremadura, concretamente del V Plan Regional de Investigación Desarrollo Tecnológico e Innovación (2014-2017), a través del Grupo de Investigación GIMAF (referencia GR15027).
}

Artículo recibido en mayo de 2017 y aceptado en agosto de 2017

Artículo disponible en versión electrónica en la página www.revista-eea.net, ref. ə-35309 


\section{INTRODUCCIÓN}

En los últimos años, la actividad de la banca comercial en España se ha desarrollado en un entorno muy adverso. El estallido de la burbuja inmobiliaria conllevó el deterioro de la calidad de su cartera de créditos y, con ello, un elevado incremento de las tasas de morosidad. A todo ello hay que unirle la desaceleración de la economía española y la crisis de deuda soberana en Europa, con las consiguientes limitaciones de financiación en los mercados internacionales.

Con el objeto de devolver la confianza en el sistema financiero español, las autoridades españolas y europeas aprobaron el Memorando de Entendimiento (MoU) el 20 de julio de 2012, por el cual se establecía el marco del proceso de recapitalización y reestructuración del sector. De este modo, las entidades financieras han visto comprometidos sus objetivos de crecimiento y rentabilidad a favor del cumplimiento de las exigencias de capital acordadas en dicho memorando.

En este contexto, el objetivo del presente estudio consiste en analizar las variables explicativas del crecimiento y la rentabilidad de la banca comercial española durante el periodo 2005-2014 teniendo en cuenta la interrelación entre ambas magnitudes así como los importantes ajustes sufridos por el sector en los últimos años.

Las aportaciones del presente estudio con respecto a la literatura previa son varias. En primer lugar, es preciso indicar que el estudio del crecimiento y la rentabilidad de la banca se ha realizado tradicionalmente por separado, ${ }^{1}$ siendo muy pocos los trabajos empíricos que han examinado la interrelación entre ambas variables (Goddard et al., 2004b; Shehzad et al., 2013; Ozacat y Tunay, 2014) sin llegar a resultados concluyentes. En este sentido consideramos que es importante aumentar la evidencia empírica internacional con nuevas bases de datos que permitan aportar nuevos resultados que contribuyan a la búsqueda de una explicación coherente y unificada acerca de cómo es esta relación.

En segundo lugar, los estudios previos sólo contemplan una relación lineal entre crecimiento y rentabilidad ignorando la posible existencia de una relación no lineal más compleja así como el periodo de crisis financiera internacional, ya que fueron realizados con anterioridad a la misma, y aún menos el impacto de los procesos de recapitalización y transferencia de los activos sobre el crecimiento y la rentabilidad en el caso concreto de la banca española. En este sentido, analizamos la posible existencia de una relación cuadrática entre ambas variables así como la influencia directa sobre el crecimiento y la rentabilidad de

${ }^{1}$ Destacando los trabajos de Wilson y Williams (2000), Goddard et al. (2002) y Homma et al. (2014) en el análisis del crecimiento y los de Demsetz y Straham (1997), Hughes et al. (1999) y Goddard et al. (2004a, 2011) en el análisis de la rentabilidad. 
las dos medidas adoptadas por las autoridades bancarias en el procesos de reestructuración y recapitalización de los bancos españoles, como son: i) la transmisión de los activos tóxicos a la Sociedad de Activos Procedentes de la Reestructuración Bancaria (SAREB) creada a tal efecto para el saneamiento y viabilidad de las entidades; ii) la concesión de ayudas públicas a determinadas entidades con necesidades de capital para poder alcanzar los niveles de solvencia exigidos por el Banco Central Europeo siguiendo los Acuerdos de Basilea III.

Los resultados obtenidos de la presente investigación nos permiten afirmar que existen evidencias de una relación cuadrática entre el crecimiento y la rentabilidad de la banca comercial en España durante el periodo de estudio. Adicionalmente, observamos que la transferencia de activos a la SAREB y las ayudas de capital para la recapitalización de determinadas entidades han tenido un efecto significativo sobre el crecimiento y la rentabilidad de las entidades analizadas.

El resto del trabajo se estructura como se describe a continuación. En el apartado segundo se presenta una revisión de la literatura previa sobre crecimiento y rentabilidad en la banca. En el apartado tercero se describen las características de la muestra. En el cuarto apartado se presenta la metodología empleada. En el quinto apartado se describen los resultados empíricos obtenidos. Por último, en el sexto apartado se presentan las conclusiones obtenidas del conjunto del trabajo.

\section{LITERATURA PREVIA}

El análisis del crecimiento y la rentabilidad en la banca han centrado el interés de numerosos investigadores con la finalidad de conocer cómo alcanzar tales objetivos. Sin embargo, el crecimiento y la rentabilidad se han estudiado tradicionalmente por separado, siendo muy pocos los trabajos que han examinado la interrelación y, por tanto, la posible existencia de bidireccionalidad entre ambos. Es por ello que el presente apartado ha sido subdividido en estas tres áreas de estudio.

\subsection{Estudios sobre el crecimiento en la banca}

El estudio empírico del crecimiento en la banca, al igual que el crecimiento empresarial, se ha ligado tradicionalmente a la ley enunciada por Gibrat (1931) que considera el crecimiento empresarial como un proceso estocástico que tiende a la concentración de los mercados. La razón de su uso, es que dicha ley considera que la distribución del tamaño se asemeja a una distribución lognormal, ya que cada entidad es objeto de una serie de shocks puramente aleatorios que permite analizar empíricamente el crecimiento en relación al tamaño. 
Existen diversos trabajos que analizan el crecimiento de la banca en diferentes países contrastando la ley de Gibrat. Entre los trabajos más destacados (Alhadeff y Alhadeff, 1964: Rhoades y Yeats, 1974; Yeats et al., 1975; Tschoegl, 1983; Wilson y Williams, 2000) cabe señalar que en la mayoría de los casos rechazan el cumplimiento de la ley de Gibrat. De este modo, es difícil creer que el crecimiento sea un proceso puramente aleatorio, siendo más probable que el crecimiento sea determinado por algún factor económico (Homma et al., 2014).

Por lo tanto, otros estudios incorporan la rentabilidad como variable explicativa del crecimiento para las entidades financieras, entre los que destaca el trabajo realizado por Goddard et al. (2002) para cooperativas de crédito en Estados Unidos durante 1990-1999, encontrando que la rentabilidad influye positivamente en el crecimiento, y hallando además una relación negativa entre tamaño y crecimiento y la no persistencia del crecimiento.

No obstante, otros aspectos que influyen en la relación tamaño-crecimiento de las entidades son los diferentes escenarios de liberalización, desregulación y procesos de integración del sector bancario. Concretamente, Benito (2008) contrastó la ley de Gibrat para el conjunto de entidades de crédito españolas durante el periodo 1970-2006 encontrando diferentes relaciones según los procesos de liberalización y consolidación. Los resultados obtenidos mostraban que cuando el sector bancario español era altamente regulado, las entidades pequeñas crecían más que las de mayor tamaño, sin embargo en los últimos años del periodo analizado encuentra que las entidades de mayor tamaño crecen a tasas superiores a las pequeñas debido a las economías de escala y a la mejora de la eficiencia.

Por último, es preciso tener en cuenta los trabajos de Goddard et al. (2014, 2016). Concretamente, Goddard et al. (2014) analizan el impacto generado por los procesos de adquisiciones y fusiones en la distribución del tamaño de las entidades de crédito de Estados Unidos durante el periodo 1994-2010. Posteriormente, Goddard et al. (2016) analizan la influencia de los cambios regulatorios en materia de exigencias de capital ocurridos en el año 2000 en Estados Unidos para un conjunto de 12.046 entidades financieras mediante datos semestrales en el periodo 1994-2012.

\subsection{Estudios sobre la rentabilidad en la banca}

Durante las últimas tres décadas se han producido cambios en el sector bancario debido a la desregulación, a los cambios tecnológicos y la globalización de bienes y servicios en los mercados financieros. El desarrollo producido en el sector ha incrementado la competencia bancaria y ha provocado un incremento del número de operaciones, una mejora de la eficiencia, un incremento de la productividad, y una lucha por ofrecer mejores precios a los clientes, que se ha 
traducido en menores márgenes por operación y, por tanto, en la convergencia de las tasas de rentabilidad bancaria en todos los países (Goddard et al., 2011).

Es preciso señalar que los trabajos que analizan la rentabilidad de los grandes bancos de Estados Unidos, como los de Levonian (1993), Roland (1997), Berger et al. (2000) y Knapp et al. (2006) destacan por su metodología y aportaciones. El primero de ellos encuentra una persistencia de la rentabilidad menor en las entidades financieras que la que se encontraban tradicionalmente para empresas industriales. El segundo introduce la utilización de datos trimestrales, el tercero hace un análisis no paramétrico encontrando diferencias entre bancos situados en los primeros y últimos deciles en función de la distribución de los bancos por su rendimiento. El cuarto y último muestra que para las entidades americanas las tasas de rentabilidad convergen a los cinco años.

Trabajos más recientes son los realizados por Goddard et al. (2004a, 2011), Trujillo (2013) y Dietrich et al. (2014). El primero de ellos se centra en el estudio de la rentabilidad de los principales bancos europeos, encontrando que la persistencia es mayor en cajas y cooperativas de crédito que en la banca comercial. El segundo trabajo, contrasta la persistencia en bancos de 65 países desarrollados encontrando evidencia de influencia negativa del crecimiento en la rentabilidad, ya que en un entorno de fuerte competencia, la persistencia tiende a ser débil en aquellas economías donde el desarrollo institucional es más avanzado y los mecanismos externos de gobierno son fuertes. El trabajo de Trujillo (2013) analiza bancos comerciales y cajas de ahorro en España en el periodo 1999-2009. En cuanto a los resultados obtenidos, se observa que ni el tamaño ni las tasas de crecimiento eran variables explicativas de la rentabilidad, mientras que el ratio de capital explicaba de forma positiva las tasas de rentabilidad. Por último, Dietrich et al. (2014) analizan cómo afecta a la rentabilidad de la banca de países centroeuropeos la influencia de las nuevas exigencias de capital establecidas en los Acuerdos de Basilea III, encontrando que sólo algunos grandes bancos se han visto afectados negativamente.

\subsection{La interrelación crecimiento-rentabilidad en la banca}

A pesar de ser el crecimiento y la rentabilidad los principales objetivos empresariales, son limitados los trabajos que examinan la interrelación entre ambos. En el ámbito empresarial destacan los trabajos de Cowling (2004), Coad (2007, 2010), Coad et al. (2011), Jang y Park (2011) y Lee (2014). En el ámbito de la banca la evidencia empírica previa que analiza esta relación se reduce a los trabajos de Goddard et al. (2004b), Shehzad et al. (2013) y Ozacat y Tunay (2014). El primero de ellos estudia la interrelación entre ambas magnitudes en los cinco mayores países de la Unión Europea (Francia, Alemania, Italia, Reino Unido y España) para el periodo 1992-1998. Para ello proponen un modelo dinámico de datos de panel estimado mediante regresiones de sección cruzada. 
Además, analizan el crecimiento y la rentabilidad bancaria en función del tamaño. De este modo, contrastan la ley de Gibrat y la hipótesis de persistencia de la rentabilidad, ya que ambas magnitudes tienden a converger en el largo plazo.

Los resultados del trabajo de Goddard et al. (2004b) no muestran evidencia de interrelación, pero sí una influencia positiva de la rentabilidad sobre el crecimiento, ya que un incremento de la rentabilidad genera beneficios, que son la fuente de financiación principal para fomentar el crecimiento. De otro modo, encuentran que un exceso del crecimiento actual puede tener consecuencias adversas, aunque no de forma significativa sobre la rentabilidad futura, debido a la existencia de limitaciones en la capacidad directiva para mantener las mismas tasas de rentabilidad ante un crecimiento excesivo.

Asimismo, el trabajo de Shehzad et al. (2013) tiene como punto de partida el trabajo de Goddard et al. (2004b) aunque utiliza una muestra más amplia compuesta de bancos de 148 países durante el periodo 1998-2010, agrupando los países por su pertenencia o no a la OCDE. Para analizar el comportamiento del crecimiento y la rentabilidad, Shehzad et al. (2013) utilizan la metodología de datos de panel estimando el modelo de Goddard et al. (2004b) mediante el Método Generalizado de los Momentos (GMM) usando el estimador de Blundell y Bond (1998) ya que aporta resultados más consistentes e insesgados que el estimador de Arellano y Bond (1991) cuando la muestra está compuesta por un número limitado de periodos de análisis y un elevado número de bancos.

Los resultados obtenidos por Shehzad et al. (2013) son similares a los obtenidos previamente por Goddard et al. (2004b) en cuanto al estudio de la rentabilidad, aceptando sin embargo la independencia del crecimiento en la banca y, por lo tanto, el cumplimiento de la ley de Gibrat ya que el crecimiento sigue un proceso aleatorio.

Por otro lado, el trabajo de Ozacat y Tunay (2014) analiza la interrelación crecimiento-rentabilidad en la banca de un país emergente, como es el caso de Turquía, para un grupo de 30 entidades financieras durante el periodo 2002-2012. Los resultados que obtienen muestran que existe una interrelación negativa en ambos sentidos entre crecimiento y rentabilidad. Además, encuentran que la rentabilidad es persistente entre periodos consecutivos y que las entidades más pequeñas son las más rentables y competitivas.

\section{BASE DE DATOS}

Para la realización del presente estudio se ha utilizado la base de datos Bureau Van Dijk's Bankscope, obteniendo datos anuales de un total de 49 bancos comerciales que tienen su actividad en España durante el periodo 2005- 
2014. Se han analizado desde grandes bancos españoles como Banco Santander o BBVA hasta bancos de menor tamaño como Banca Pueyo o Banca March. ${ }^{2}$

Por otro lado, siguiendo los trabajos de Goddard et al. (2004a, 2004b), el crecimiento de las entidades financieras ha sido calculado a partir de la diferencia en la cifra de activos entre dos periodos consecutivos mientras que la rentabilidad de los activos totales ha sido la medida de rentabilidad empleada.

El tamaño de las entidades financieras, medido en base a su cifra de activos, ha sido empleado como variable de control por sus implicaciones en el crecimiento y en la rentabilidad empresarial como se ha observado en la literatura previa. Adicionalmente, hay que tener en cuenta que la banca ha respondido a la concentración y la presión de la competencia ampliando su gama de servicios y productos financieros e incrementando su porcentaje de negocio fuera de balance. De esta manera las entidades han conseguido mejorar la diversificación de sus carteras y niveles de riesgo. Es por ello que también incluimos en nuestro estudio empírico una variable de control denominada Off Balance Sheet que representa los servicios de gestión de productos e instrumentos derivados fuera de la operativa corriente.

Por último, siguiendo a Goddard et al. (2004b), también incluimos como variables de control los ratios de capital y liquidez para analizar si la calidad crediticia de una entidad influye en la rentabilidad de la misma. Como estos autores señalan, un exceso de capital podría significar que un banco es conservador en su actividad haciendo caso omiso de la posible rentabilidad que le podría proporcionar una mayor diversificación del negocio bancario. Por otro lado, también consideran que un banco con una elevada proporción de activos líquidos es poco probable que obtenga altas tasas de rentabilidad.

Los estadísticos descriptivos y la matriz de correlaciones de las variables objeto de estudio se presentan en la Tabla 1. En relación con los estadísticos descriptivos es preciso indicar que las entidades en España han crecido de media un $4,37 \%$ y han alcanzado una rentabilidad media del 0,45\%, presentando un nivel medio de ratio de capital del 17,24\%. La diversificación media de la cartera de productos OBS es de un $0,61 \%$, además son bajos pero positivos los niveles de liquidez y la tasa de morosidad media del 5,74\% y $3,24 \%$, respectivamente. Por otro lado, la matriz de correlaciones muestra que el crecimiento y la rentabilidad están poco correlacionados, siendo la influencia del tamaño prácticamente nula respecto al crecimiento y la rentabilidad, siendo

\footnotetext{
${ }^{2}$ Es preciso también indicar que el panel de datos formado no está balanceado debido a que incluye el alta y el cese de la actividad de entidades debido al proceso de reestructuración del sector durante el periodo analizado. Concretamente, en el caso de fusiones, consideramos que la entidad creada es una nueva entidad en la base de datos, mientras que en el caso de absorciones, las entidades absorbidas consideramos que cesan su actividad continuando la de la entidad que las ha absorbido.
} 
negativa la influencia de las tasas de morosidad sobre las variables objeto de estudio. Por lo tanto, existe poco riesgo de multicolinealidad en los modelos que se plantean en el trabajo ya que las variables endógenas que se usan no presentan altas correlaciones entre sí.

Tabla 1

Estadísticos descriptivos y matriz de correlaciones

\begin{tabular}{|c|c|c|c|c|c|c|c|}
\hline \multicolumn{8}{|c|}{ Estadísticos Descriptivos } \\
\hline & Crec & Rent & Tam & Liq & Cap & Mor & Obs \\
\hline Media & 4,37 & 0,45 & 15,61 & 5,74 & 17,24 & 3,24 & 0,61 \\
\hline Mediana & 2,71 & 0,44 & 15,34 & 4,94 & 7,62 & 2,27 & 0,10 \\
\hline Máximo & 65,04 & 11,01 & 20,96 & 33,61 & 100,00 & 18,86 & 6,59 \\
\hline Mínimo & $-72,11$ & $-12,01$ & 9,44 & 0,00 & $-5,95$ & 0,00 & 0,00 \\
\hline Desv. Stand. & 17,00 & 1,96 & 2,78 & 4,76 & 25,16 & 3,56 & 1,16 \\
\hline \multicolumn{8}{|c|}{ Matriz de correlaciones } \\
\hline & Crec & Rent & Tam & Liq & Cap & Mor & Obs \\
\hline Crec & 1,00 & & & & & & \\
\hline Rent & 0,35 & 1,00 & & & & & \\
\hline Tam & $-0,00$ & 0,03 & 1,00 & & & & \\
\hline Liq & 0,07 & $-0,03$ & 0,39 & 1,00 & & & \\
\hline Cap & $-0,20$ & 0,11 & $-0,44$ & $-0,01$ & 1,00 & & \\
\hline Mor & $-0,26$ & $-0,25$ & 0,31 & 0,38 & $-0,20$ & 1,00 & \\
\hline Obs & $-0,12$ & $-0,05$ & $-0,05$ & $-0,04$ & 0,13 & 0,25 & 1,00 \\
\hline
\end{tabular}

La Tabla representa los estadísticos descriptivos y matriz de correlaciones de las variables crecimiento Crec, rentabilidad Rent, tamaño Tam, ratio de capital Cap, diversificación Obs, liquidez Liq, morosidad Mor. En el contraste aumentado de Dickey-Fuller el número de retardos óptimo está determinado siguiendo el criterio de Akaike mientras que para los contrastes PP se sigue el procedimiento de Newey y West. En todos los casos los contrastes responden a la utilización de una ecuación auto regresiva que solo incluye el término constante.

***, ** $y$ * supone el rechazo de la hipótesis nula al 1\%, 5\% y 10\% de nivel de significatividad respectivamente.

Fuente: Elaboración propia.

Por último, es preciso señalar que se ha realizado un análisis de vectores autorregresivos así como el test de causalidad de Granger que nos confirman que existe una influencia significativa del crecimiento y la rentabilidad del año $t-1$ sobre el crecimiento y rentabilidad del año $t$.

\section{METODOLOGÍA}

En este apartado se presentan los modelos lineales y no lineales utilizados para examinar el crecimiento y la rentabilidad de la banca en España. Para ello, empleamos la metodología de datos de panel. Esta metodología permite modelizar la heterogeneidad inobservable que existe entre las distintas empresas mediante la descomposición del término de error en tres componentes, permitiendo además aumentar el número de observaciones, los grados de libertad de los modelos y por tanto la consistencia de los resultados. 
En primer lugar, se utilizan los modelos lineales dinámicos multivariantes planteados por Goddard et al. (2004b) y utilizados por Shehzad et al. (2013) para analizar el crecimiento y la rentabilidad en la banca, que son expresados del siguiente modo:

$$
\begin{aligned}
& \operatorname{Crec}_{i, t}=\beta_{0}+\beta_{1} \text { Crec }_{i, t-1}+\beta_{2} \text { Rent }_{i, t-1}+\beta_{3} \text { Tam }_{i, t-1}+\sum_{i=4}^{7} \beta_{k} V C_{i, t-1}+\mu_{i, t} \\
& \text { Rent }_{i, t}=\beta_{0}+\beta_{1} \text { Rent }_{i, t-1}+\beta_{2} \text { Crec }_{i, t-1}+\beta_{3} \text { Tam }_{i, t-1}+\sum_{i=4}^{7} \beta_{k} V C_{i, t-1}+\mu_{i, t}
\end{aligned}
$$

donde $\operatorname{Crec}_{i, t}$ y Rent $_{i, t}$ representa el crecimiento y la rentabilidad del banco $i$ en el momento $t$ respectivamente, que vienen explicados por las variables endógenas retardadas un periodo del crecimiento y la rentabilidad, ${ }^{3}$ además de un conjunto de variables de control que representan el tamaño, el nivel de solvencia, la tasa de liquidez, la tasa de morosidad y el porcentaje del negocio fuera del balance. Por último, $\beta_{k}$ son los parámetros a estimar y $\mu_{i, t}$ el término de error. ${ }^{4}$

No obstante, estudios previos realizados para entidades no financieras han encontrado evidencia empírica de relación positiva y negativa entre los objetivos empresariales de crecimiento y rentabilidad. En este sentido, Lee (2014) expone que la relación entre crecimiento y rentabilidad empresarial es una cuestión empírica, ya que de la literatura no se puede extraer un resultado concluyente. Por tanto, considera que una relación no lineal entre estas dos variables podría recoger tanto la relación positiva que consideran algunos autores como la negativa que argumentan otros cuando se alcanzan tasas de crecimiento elevadas. De otro modo, las teorías gerenciales evidenciaban la posibilidad de una relación cuadrática en forma de U invertida, ya que la influencia positiva del crecimiento sobre la rentabilidad podría volverse negativa si el crecimiento fuese tan elevado que provocase dificultades en la organización empresarial, invirtiéndose el sentido de la relación. Teniendo en cuenta estos aspectos, proponemos los siguientes modelos para examinar la posible existencia de relación no lineal entre crecimiento y rentabilidad en la banca comercial española:

\footnotetext{
${ }^{3}$ Siguiendo la literatura previa, se asume que existe causalidad entre el crecimiento y la rentabilidad, por lo tanto, puede surgir un posible problema de endogeneidad que debe ser abordado. Es por ello que en los modelos propuestos, las variables explicativas son introducidas retardadas un periodo para mitigar dicho problema de endogeneidad.

${ }^{4}$ Concretamente, $u_{i, t}=\eta_{i}+\lambda_{t}+v_{k}$ donde $\eta_{i}$ es la parte del error específica de cada unidad (heterogeneidad inobservable) y que recoge aquellos efectos no observables que afectan solo a la entidad $i, \lambda_{t}$ representa aquellos shocks que ocurren en el momento $t \mathrm{y}$ afectan a todos las entidades por igual y $v_{k}$ es una perturbación aleatoria.
} 


$$
\begin{aligned}
& \operatorname{Crec}_{i, t}=\beta_{0}+\beta_{1} \operatorname{Crec}_{i, t-1}+\beta_{2} \operatorname{Rent}_{i, t-1}+\beta_{3} \operatorname{Rent}_{i, t-1}^{2}+\beta_{4} \operatorname{Tam}_{i, t-1}+\sum_{k=5}^{8} V C_{i, t-1}+\mu_{i, t} \\
& \operatorname{Rent}_{i, t}=\beta_{0}+\beta_{1} \operatorname{Rent}_{i, t-1}+\beta_{2} \operatorname{Crec}_{i, t-1}+\beta_{3} \operatorname{Crec}_{i, t-1}^{2}+\beta_{4} \operatorname{Tam}_{i, t-1}+\sum_{k=5}^{8} V C_{i, t-1}+\mu_{i, t}
\end{aligned}
$$

donde $\operatorname{Crec}^{2}$ y Rent $^{2}$ son las variables cuadráticas de crecimiento y rentabilidad respectivamente.

Por último, consideramos conveniente analizar la influencia directa sobre el crecimiento y la rentabilidad de las medidas adoptadas por las autoridades bancarias para favorecer el saneamiento y viabilidad de los bancos españoles, bajo en marco del MoU. Concretamente, analizamos el efecto de la transmisión de los activos más problemáticos a la SAREB, así como de las ayudas públicas otorgadas a determinadas entidades con necesidades de capital para que pudieran alcanzar los niveles de solvencia exigidos por el Banco Central Europeo siguiendo los Acuerdos de Basilea III.

Para realizar dicho análisis, incluimos en los modelos no lineales anteriores las variables Sareb y Ayudas en los modelos de crecimiento y rentabilidad, respectivamente, quedando los modelos del siguiente modo:

$$
\begin{aligned}
& \operatorname{Crec}_{i, t}=\beta_{0}+\beta_{1} \text { Crec }_{i, t-1}+\beta_{2} \text { Rent }_{i, t-1}+\beta_{3} \text { Rent }_{i, t-1}^{2}+\beta_{4} \text { Sareb } \beta_{5} \text { Tam }_{i, t-1}+\sum_{k=6}^{9} V C_{i, t-1}+\mu_{i, t} \\
& \text { Rent }_{i, t}=\beta_{0}+\beta_{1} \text { Rent }_{i, t-1}+\beta_{2} \text { Crec }_{i, t-1}+\beta_{3} \text { Crec }_{i, t-1}^{2}+\beta_{4} \text { Ayudas }+\beta_{5} \text { Tam }_{i, t-1}+\sum_{K=6}^{9} V C_{i, t-1}+\mu_{i, t}
\end{aligned}
$$

donde la variable denominada Sareb incluye el valor de los activos que cada una de las entidades financieras cedió a dicha entidad en 2013, mientras la variable Ayudas representa las ayudas públicas de capital que han recibido algunas entidades financieras durante el proceso de reestructuración y recapitalización, siendo éstas contabilizadas en 2012. ${ }^{5}$

Por otro lado, es preciso indicar que el método elegido para obtener estimaciones robustas y eficientes es el Método Generalizado de los Momentos (GMM) de diferencias (Arellano y Bond, 1991) que se trata de un método particular de variables instrumentales. En el caso de que la variable dependiente sea persistente ${ }^{6}$ se utiliza el estimador de sistemas del Método Generalizado de los Momentos (GMM) propuesto por Blundell y Bond (1998).

\footnotetext{
${ }^{5}$ En un anexo aparecen las cuantías de las variables Sareb y Ayudas por entidades.

${ }^{6}$ Se considera que la variable dependiente es persistente cuando el valor del coeficiente de la variable dependiente retardada es próximo a 1.
} 
Los instrumentos utilizados para solucionar el problema de endogeneidad no deberán estar correlacionados con el término de error, pero deberán tener la capacidad de aportar la información sobre la variable explicativa. La selección de los retardos se ha realizado usando el criterio de Hannan-Quin, siendo uno el número de retardos seleccionados, ya que un mayor número de retardos no proporciona más información al modelo coincidiendo además con el trabajo realizado por Goddard et al. (2004b).

Para mostrar la significatividad global del modelo presentamos los resultados del test de Wald expresado por el estadístico $F$ para comprobar la significación conjunta de los coeficientes de las variables explicativas. También presentamos el estadístico $Z_{1}$ para comprobar la significación conjunta de las variables interaccionadas de manera que se pueda verificar que los efectos totales son efectivamente significativos. Por otro lado, para comprobar la validez de los instrumentos se usa el test de Hansen de sobreidentificación de los instrumentos. Por último, se realiza el test de Arellano y Bond (1991) para comprobar la ausencia de autocorrelación de segundo orden expresado mediante el estadístico $m_{2 .}$

\section{RESULTADOS EMPÍRICOS}

En este apartado se presentan los resultados obtenidos del conjunto de análisis efectuados. En primer lugar, se muestran los resultados del análisis del crecimiento y la rentabilidad mediante un modelo dinámico lineal. En segundo lugar, se presentan los resultados del estudio de la relación no lineal entre crecimiento y rentabilidad. En tercer lugar, se presentan los resultados obtenidos tras analizar el impacto directo sobre el crecimiento y la rentabilidad respectivamente de la transferencia de activos tóxicos a la SAREB así como de la asistencia financiera recibida por determinadas entidades con necesidades de capital.

Concretamente, en la Tabla 2 presentamos los resultados obtenidos del análisis lineal del crecimiento y la rentabilidad. En primer lugar, la influencia de la rentabilidad sobre el crecimiento es significativa y de signo negativo, en contra de la persistencia del crecimiento entre años consecutivos. Por otro lado, atendiendo a la influencia de las variables de control sobre el crecimiento, cabe destacar que las entidades que más crecen son aquellas que tienen un mayor ratio de capital y una menor liquidez.

En la Tabla 2 también se presentan los resultados relativos al análisis de la rentabilidad. En líneas generales se observa que la rentabilidad de las entidades financieras es explicada de manera positiva y significativa por las tasas de crecimiento y por la ratio de capital y, de manera negativa, por la influencia del tamaño y de la morosidad de las entidades. El conjunto de los resultados que se muestran en la Tabla 3 sugieren la existencia de interrelación entre crecimiento 
y rentabilidad aunque de distinto signo. No obstante, como hemos señalado anteriormente, esa relación puede ser más compleja. Por ello realizamos un análisis no lineal de la relación crecimiento-rentabilidad.

Tabla 2

Análisis lineal del crecimiento y de la rentabilidad

\begin{tabular}{|c|c|c|c|}
\hline & Crecimiento & & Rentabilidad \\
\hline Crecimiento (-1) & $\begin{array}{c}0,128^{\star *} \\
(2,35)\end{array}$ & Rentabilidad (-1) & $\begin{array}{c}-0,248^{\star \star \star} \\
(-2,93)\end{array}$ \\
\hline Rentabilidad (-1) & $\begin{array}{c}-4,399^{\star *} \\
(-2,66)\end{array}$ & Crecimiento (-1) & $\begin{array}{c}0,035^{\star \star \star} \\
(5,35)\end{array}$ \\
\hline Tamaño (-1) & $\begin{array}{l}9,104 \\
(0,52)\end{array}$ & Tamaño (-1) & $\begin{array}{c}-2,422^{\star \star \star} \\
(-3,14)\end{array}$ \\
\hline Liquidez (-1) & $\begin{array}{c}-1,343^{\star \star} \\
(-2,26)\end{array}$ & Liquidez (-1) & $\begin{array}{l}0,052 \\
(0,86)\end{array}$ \\
\hline R. Capital (-1) & $\begin{array}{c}5,271^{\star \star \star} \\
(4,83)\end{array}$ & R. Capital (-1) & $\begin{array}{c}0,153^{\star *} \\
(2,06)\end{array}$ \\
\hline Morosidad (-1) & $\begin{array}{l}-1,795 \\
(-0,98)\end{array}$ & Morosidad (-1) & $\begin{array}{c}-0,134^{\star \star} \\
(-2,04)\end{array}$ \\
\hline Off balance sheet (-1) & $\begin{array}{l}-2,662 \\
(-0,48)\end{array}$ & Off balance sheet $(-1)$ & $\begin{array}{l}0,011 \\
(0,10)\end{array}$ \\
\hline $\mathrm{F}$ & $29,584^{\star \star \star}$ & $\mathrm{F}$ & $5,776^{\star \star \star}$ \\
\hline $\mathrm{m}_{2}$ & 0,004 & $\mathrm{~m}_{2}$ & 0,127 \\
\hline Hansen & 5,647 & Hansen & 55,901 \\
\hline
\end{tabular}

En la tabla se recogen los resultados de la estimación de los modelos lineales de crecimiento y rentabilidad estimados mediante Método Generalizado de los Momentos (GMM). Las variables independientes se introducen con un retardo para evitar problemas de endogeneidad según el criterio de Hannan-Quin (crecimiento, rentabilidad, tamaño, ratio de capital, ratio de morosidad, liquidez y porcentaje de activos fuera de balance). Por último, presentamos los test de buen ajuste del modelo mediante el test de Wald representado por la $F$ de Snedecor, el test $m_{2}$ de no existencia de autocorrelación de segundo orden y el test de Hansen de sobreidentificación de los instrumentos.

${ }^{* * *},{ }^{* *} y$ * supone el rechazo de la hipótesis nula al 1\%, 5\% y 10\% respectivamente.

Fuente: Elaboración propia.

Los resultados del análisis no lineal se presentan en la Tabla 3. En primer lugar presentamos los resultados obtenidos en el estudio del crecimiento. Al igual que observábamos en el análisis lineal, los resultados de este nuevo análisis nos indican que la influencia de la rentabilidad sobre el crecimiento es significativa y de signo negativo. En cambio, los resultados relativos al análisis de la rentabilidad nos indican que el crecimiento de las entidades influye de forma no lineal en forma de U invertida, influyendo positivamente sobre la rentabilidad hasta alcanzar un tamaño óptimo en el que un exceso de crecimiento influye de manera negativa sobre la rentabilidad. Entre el resto de resultados destaca la influencia significativa y positiva del ratio de capital sobre las variables de estudio, en contra de la influencia negativa que ejerce la morosidad sobre dichas variables. 
Tabla 3

Análisis no lineal del crecimiento y de la rentabilidad

\begin{tabular}{|c|c|c|c|}
\hline & Crecimiento & & Rentabilidad \\
\hline Crecimiento (-1) & $\begin{array}{l}-0,075 \\
(-1,01)\end{array}$ & Rentabilidad (-1) & $\begin{array}{c}-0,214^{\star \star \star} \\
(-5,28)\end{array}$ \\
\hline Rentabilidad (-1) & $\begin{array}{c}-5,682^{\star \star} \\
(-2,51)\end{array}$ & Crecimiento $(-1)$ & $\begin{array}{c}0,040^{\star \star \star} \\
(6,62)\end{array}$ \\
\hline Rentabilidad $^{2}(-1)$ & $\begin{array}{l}-0,235 \\
(-0,68)\end{array}$ & Crecimiento $^{2}(-1)$ & $\begin{array}{c}-0,001^{\star \star *} \\
(-4,98)\end{array}$ \\
\hline Tamaño (-1) & $\begin{array}{c}13,869 * \\
(1,78)\end{array}$ & Tamaño (-1) & $\begin{array}{c}-1,286^{\star \star *} \\
(-2,93)\end{array}$ \\
\hline Liquidez (-1) & $\begin{array}{c}-2,135^{\star \star \star} \\
(-3,36)\end{array}$ & Liquidez (-1) & $\begin{array}{c}0,048^{\star} \\
(1,69)\end{array}$ \\
\hline R. Capital (-1) & $\begin{array}{c}5,286^{\star \star \star} \\
(11,58)\end{array}$ & R. Capital (-1) & $\begin{array}{c}0,175^{\star \star \star} \\
(3,66)\end{array}$ \\
\hline Morosidad (-1) & $\begin{array}{c}-1,914^{\star \star} \\
(-2,05)\end{array}$ & Morosidad (-1) & $\begin{array}{c}-0,109 * * * \\
(-2,70)\end{array}$ \\
\hline Off balance sheet $(-1)$ & $\begin{array}{l}-0,782 \\
(-0,82) \\
\end{array}$ & Off balance sheet $(-1)$ & $\begin{array}{l}0,005 \\
(0,15) \\
\end{array}$ \\
\hline $\mathrm{F}$ & $36,744^{\star \star \star}$ & $\mathrm{F}$ & $7,635^{\star \star \star}$ \\
\hline $\mathrm{m}_{2}$ & $-0,249$ & $\mathrm{~m}_{2}$ & 0,080 \\
\hline Hansen & 35,955 & Hansen & 32,036 \\
\hline
\end{tabular}

En la tabla se recogen los resultados de la estimación de los modelos lineales de crecimiento y rentabilidad estimados mediante Método Generalizado de los Momentos (GMM). Las variables independientes se introducen con un retardo para evitar problemas de endogeneidad según el criterio de Hannan-Quin (crecimiento, rentabilidad, tamaño, ratio de capital, ratio de morosidad, liquidez y porcentaje de activos fuera de balance). Por último, presentamos los test de buen ajuste del modelo mediante el test de Wald representado por la $\mathrm{F}$ de Snedecor, el test $m_{2}$ de no existencia de autocorrelación de segundo orden y el test de Hansen de sobreidentificación de los instrumentos.

${ }^{* * *},{ }^{* \star} y$ * supone el rechazo de la hipótesis nula al 1\%, 5\% y 10\% respectivamente.

Fuente: Elaboración propia.

Por último, como ya indicamos en el apartado metodológico, resulta interesante analizar la influencia que ha tenido sobre el crecimiento el saneamiento de los balances mediante la transferencia de los activos tóxicos al "banco malo" y efecto sobre la rentabilidad de las ayudas públicas otorgadas a determinadas instituciones, observándose los resultados de dichos análisis en la Tabla 4. Los resultados obtenidos muestran que la asistencia financiera ha tenido un efecto positivo sobre la rentabilidad de las entidades manteniendo los resultados para el resto de variables. Por otro lado, la transferencia de activos tóxicos a la SAREB tiene una influencia negativa y significativa sobre el crecimiento de las entidades, lo que supone una reducción del tamaño de las entidades, aunque se considera que la salida de activos tóxicos puede ayudar a la mejora de la capacidad crediticia de las entidades cedentes. 
Tabla 4

Efecto de la recapitalización y transferencia de activos a la Sareb

\begin{tabular}{|c|c|c|c|}
\hline & Crecimiento & & Rentabilidad \\
\hline Crecimiento (-1) & $\begin{array}{l}-0,072 \\
(-0,92)\end{array}$ & Rentabilidad (-1) & $\begin{array}{c}-0,262^{\star \star} \\
(-2,59)\end{array}$ \\
\hline Rentabilidad (-1) & $\begin{array}{c}-8,490 * \star \star \\
(-3,49)\end{array}$ & Crecimiento (-1) & $\begin{array}{c}0,044^{\star \star *} \\
(5,87)\end{array}$ \\
\hline Rentabilidad $^{2}(-1)$ & $\begin{array}{c}-0,698^{\star} \\
(-1,91)\end{array}$ & Crecimiento $^{2}(-1)$ & $\begin{array}{c}-0,001^{\star \star *} \\
(-4,03)\end{array}$ \\
\hline Tamaño (-1) & $\begin{array}{c}21,400^{\star \star \star} \\
(2,62)\end{array}$ & Tamaño (-1) & $\begin{array}{l}-0,624 \\
(-0,92)\end{array}$ \\
\hline Liquidez (-1) & $\begin{array}{c}-1,687^{\star \star \star} \\
(-4,50)\end{array}$ & Liquidez $(-1)$ & $\begin{array}{l}0,044 \\
(0,83)\end{array}$ \\
\hline R. Capital (-1) & $\begin{array}{c}5,351^{\star \star \star} \\
(10,82)\end{array}$ & R. Capital (-1) & $\begin{array}{c}0,221^{\star \star \star} \\
(2,77)\end{array}$ \\
\hline Morosidad (-1) & $\begin{array}{c}-3,338^{\star \star *} \\
(-5,15)\end{array}$ & Morosidad (-1) & $\begin{array}{l}-0,116^{\star} \\
(-1,93)\end{array}$ \\
\hline Off balance sheet $(-1)$ & $\begin{array}{c}-2,624^{\star \star \star} \\
(-3,27)\end{array}$ & Off balance sheet $(-1)$ & $\begin{array}{l}-0,051 \\
(-0,47)\end{array}$ \\
\hline SAREB & $\begin{array}{c}-0,001^{\star \star *} \\
(-4,52)\end{array}$ & Ayudas Públicas & $\begin{array}{c}0,001^{\star \star} \\
(2,42)\end{array}$ \\
\hline $\mathrm{F}$ & $38,562^{\star \star \star}$ & $\mathrm{F}$ & $18,124^{\star \star \star}$ \\
\hline $\mathrm{Z}_{1}$ & $3,83^{\star \star}$ & $\mathrm{Z}_{1}$ & $2,95^{\star \star}$ \\
\hline $\mathrm{m}_{2}$ & 0,76 & $\mathrm{~m}_{2}$ & 1,27 \\
\hline Hansen & 40,395 & Hansen & 53,006 \\
\hline
\end{tabular}

En la tabla se recogen los resultados de la estimación de los modelos lineales de crecimiento y rentabilidad estimados mediante Método Generalizado de los Momentos (GMM). Las variables independientes se introducen con un retardo para evitar problemas de endogeneidad según el criterio de Schwarz (crecimiento, rentabilidad, tamaño, ratio de capital, ratio de morosidad, liquidez y porcentaje de activos fuera de balance, Sareb y Ayudas Públicas). Por último, presentamos los test de buen ajuste del modelo mediante el test de Wald representado por la $F$ de Snedecor, el test $m_{2}$ de no existencia de autocorrelación de segundo orden y el test de Hansen de sobreidentificación de los instrumentos, además se incorpora $Z_{1}$ que representa el resultado del test de Wald de significación conjunta de las variables interaccionadas.

${ }^{* * *},{ }^{* *} y{ }^{*}$ supone el rechazo de la hipótesis nula al 1\%, 5\% y 10\% respectivamente.

Fuente: Elaboración propia.

\section{CONCLUSIONES}

La crisis económica y financiera, así como la de deuda soberana, han afectado a la estabilidad de la banca en España siendo necesario un proceso de reestructuración, recapitalización y de transferencia de activos inmobiliarios tóxicos. En el presente estudio se ha realizado un análisis de la interrelación entre crecimiento y rentabilidad en la banca comercial española en dicho contexto.

Las dificultades que han atravesado los bancos españoles en los últimos años hacen prever que la relación entre las variables objeto de estudio pueda ser compleja. Por ello, a diferencia de los estudios empíricos previos, en el presente trabajo se propone un análisis no lineal de la relación entre crecimiento y rentabilidad y se analiza el efecto directo de las medidas adoptadas en términos 
de saneamiento y recapitalización de los balances de dichas entidades, sin olvidar otros aspectos como el tamaño, la solvencia y la morosidad de las mismas.

Los resultados del conjunto del trabajo nos indican que existe una relación cuadrática entre crecimiento y rentabilidad para el conjunto de bancos comerciales que operan en España durante los años de estudio. En cuanto al impacto de las medidas de saneamiento y recapitalización, se ha observado que han afectado significativamente al crecimiento y a la rentabilidad.

Por último, debido a la influencia de los ratios de capital, liquidez y diversificación del negocio bancario sobre el crecimiento y la rentabilidad, consideramos que sería interesante analizar en investigaciones futuras la influencia del crecimiento y de la rentabilidad sobre los niveles de solvencia y liquidez, de manera que se pueda examinar cómo influye la banca comercial en la función de estabilidad del sector financiero español.

\section{REFERENCIAS BIBLIOGRÁFICAS}

ALHADEFF, D.A. y ALHADEFF, C.P. (1964). "Growth of Large Banks, 1930-1960". Review of Economics and Statistics, 46, pp. 356-363.

ARELLANO, M. y BOND, S. (1991). "Some tests of specification for panel data: Monte Carlo evidence and an application to employment equations". Review of Economic Studies, 58(2), pp. 277-297.

BENITO, E. (2008). "Size, growth and bank dynamics". Documento de trabajo no 0801. Banco de España.

BERGER, A.N.; BONIME, S.D.; COVITZ, D.M. y HANCOCK, D. (2000). "Why are bank profits so persistent? The roles of product market competition, informational opacity and regional/macroeconomic shocks". Journal of Banking and Finance, 24, pp. 12031235.

BLUNDELL, R. y BOND, S. (1998). "Initial conditions and moment restrictions in dynamic panel data models". Journal of Econometrics, 87, pp. 115-143.

COAD, A. (2007). "Testing the principle of 'growth of the fitter'. The relationship between profits and firm growth". Structural Change and Economic Dynamics, 18(3), pp. 370386.

COAD, A. (2010). "Exploring the processes of firm growth. Evidence from a vector autoregression". Industrial and Corporate Change, 19(6), pp. 1677-1703.

COAD, A.; RAO, R. y TAMAGNI, F. (2011). "Growth processes of Italian manufacturing firms". Structural Change and Economic Dynamics, 22(1), pp. 54-70.

COWLING, M. (2004). "The growth-profit Nexus". Small Business Economics, 22(1), pp. 1-9.

DEMSETZ, R. S. y STRAHAN P. E. (1997). "Diversification, Size, and Risk at Bank Holding Companies". Journal of Money, Credit, and Banking, 29, pp. 300-313. 
DIETRICH, A.; HESS, K. y WANZENRIED, G. (2014). "The good and bad news about the new liquidity rules of Basel III in Western European countries". Journal of Banking and Finance, 44, pp. 13-25.

GIBRAT, R. (1931). Les inegalites economiques. Paris: Librairie du Recueil Sirey.

GODDARD, J.; MCKILLOP, D.G. y WILSON, J.O.S. (2002). "The Growth of US Credit Unions". Journal of Banking and Finance, 22, pp. 2327-2356.

GODDARD, J.; MCKILLOP, D.G. y WILSON, J.O.S. (2014). "U.S. credit unions: Survival, consolidation, and growth". Economic Inquiry, 52(1), pp. 304-319.

GODDARD, J.; MCKILLOP, D.G. y WILSON, J.O.S. (2016). "Regulatory change and capital adjustment of US credit unions". Journal of Financial Service Research, 50(1), pp. 29-55.

GODDARD, J.; MOLYNEUX, P. y WILSON, J.O.S. (2004a). "The profitability of European Banks: a cross-sectional and dynamic panel analysis". The Manchester School, 72(3), pp. 363-381.

GODDARD, J.; MOLYNEUX, P. y WILSON, J.O.S. (2004b). "Dynamics of growth and profitability in banking". Journal of Money, Credit and Banking, 36(6), pp. 1069-1090.

GODDARD, J.; LIU H.; MOLYNEUX, P. y WILSON, J.O.S. (2011). "The persistence of bank profit". Journal of Banking and Finance, 35, pp. 2881-2890.

HOMMA, T.; TSUTSUI Y. y UCHIDA, H. (2014). "Firm growth and efficiency in the banking industry: A new test of the efficient structure hypothesis". Journal of Banking and Finance, 40, pp. 143-153.

HUGHES, J.P.; LANG, W.; MESTER, L. y MOON, C.G. (1999). "The Dollar and Sense of Bank Consolidation". Journal of Banking and Finance, 23, pp 291-324.

JANG, S. y PARK, K. (2011). "Inter-relationship between firm growth and profitability". International Journal of Hospitality Management, 30(4), pp. 1027-1035.

KNAPP, M.; GART, A. y CHAUDHRY, M. (2006). "The impact of persistence and mean reversion of bank profitability on post-merger performance in the banking industry". Journal of Banking and Finance, 30, pp. 3503-3517.

LEE, S. (2014). "The relationship between growth and profit: evidence from firm-level panel data". Structural Change and Economic Dynamics, 28, pp. 1-11.

LEVONIAN, M.E. (1993). "The Persistence of Bank Profits: What the Stock Market Implies". Federal Reserve Bank of San Francisco Working Papers. Applied Economic Theory, vol. 93/15.

Memorando de Entendimiento sobre condiciones de Política Sectorial Financiera, hecho en Bruselas y Madrid el 23 de julio de 2012, y Acuerdo Marco de Asistencia Financiera, hecho en Madrid y Luxemburgo el 24 de julio de 2012. BOE $n^{\circ} 296,10$ de diciembre de 2012.

OZATAC, N. y TUNAY, B. (2014). "The analysis of interaction between growth and profitability on the basis of growth persistency: the case of Turkey". American Journal of Economics, 4(6), pp. 219-225.

RHOADES, S.A. y YEATS, A.J. (1974). "Growth, consolidation and mergers in banking". Journal of Finance, 29, pp. 1397-1405.

ROLAND, K.P. (1997). "Profit Persistence in Large US Bank Holding Companies: An Empirical Investigation". Office of the Controller of Currency Working Paper 97-2.

ROODMAN, D. (2009). "How to do xtabond2: A introduction to difference and system GMM in Stata". The Stata Journal, 9(1), pp. 86-136. 
SHEHZAD, C.T.; De HAAN, J. y SHOLTENS, B. (2013). "The relationship between size, growth and profitability of commercial banks". Applied Economics, 45, pp. 17511765.

TSCHOEGL, A.E. (1983). "Size, growth and transnationality among the world's largest Banks". Journal of Business, 56, pp. 187-201.

TRUJILLO, A. (2013). "What determines the profitability of banks? Evidence from Spain". Accounting \& Finance, 53(2), pp. 561-686.

WILSON, J.O.S. y WILLIAMS, J. (2000). "The size and growth of banks: Evidence from four European countries". Applied Economics, 32, pp. 1101-1109.

YEATS, A.J.; IRONS, E.D. y RHOADES, S.A. (1975). "An analysis of new bank growth". Journal of Business, 48, pp. 199-203. 


\section{Anexo}

Valores de las variables Sareb y Ayudas por entidades

\begin{tabular}{lcc}
\hline Entidad & $\begin{array}{c}\text { Activos adquiridos por la } \\
\text { Sareb (millones de } € \text { ) }\end{array}$ & $\begin{array}{c}\text { Ayudas de capital recibidas } \\
\text { (millones de } € \text { ) }\end{array}$ \\
\hline Bankia-BFA & 22.317 & 24.743 \\
Catalunya Banc & 6.708 & 10.825 \\
NCG Banco & 5.097 & 7.176 \\
Banco Valencia & 1.962 & 3.462 \\
BMN & 5.820 & 2.208 \\
CEISS & 3.137 & 2.063 \\
Liberbank & 2.918 & 1.198 \\
Caja3 & 2.212 & 779 \\
\hline
\end{tabular}

Fuente: Elaboración propia.

En el anexo se presentan los valores de las variables Sareb y Ayudas por entidades financieras. Así, en la primera columna se pueden observar en primer lugar las entidades nacionalizadas (Bankia, Catalunya-Banc, NCG-Banco y Banco de Valencia) seguidas por BMN, CEISS, Liberbank y Caja3. En la segunda columna del anexo se detalla el valor de los activos adquiridos por la Sareb a cada una de las entidades financieras Por último, en la tercera columna se observan las ayudas de capital que recibieron las entidades durante el proceso de reestructuración y recapitalización en 2012 